\title{
Comparación del estrés oxidativo en mujeres con diabetes gestacional versus embarazos normoevolutivos
}

Terrones Saldivar Ma del Carmen*, Rodríguez-Gustavo Martin**, Rosas-Cabral Alejandro*, Góngora-Ortega Javier***, Serna-Vela Francisco**, Reyes-Robles Martha Elena***, Sandoval-Valdez Darío Alejandro****, Hernández-González Flavio ${ }^{* * * * *}$, De la Rosa-Félix Hesli Esther****, ZúñigaSánchez Karina Altagracia*****

\begin{tabular}{|c|c|}
\hline 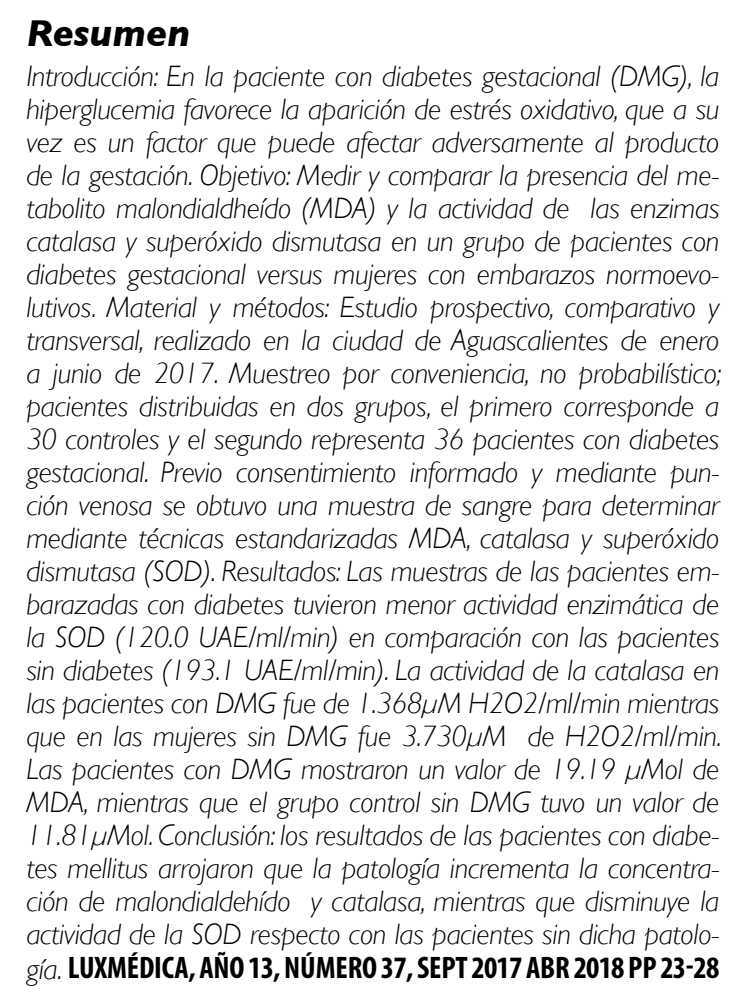 & 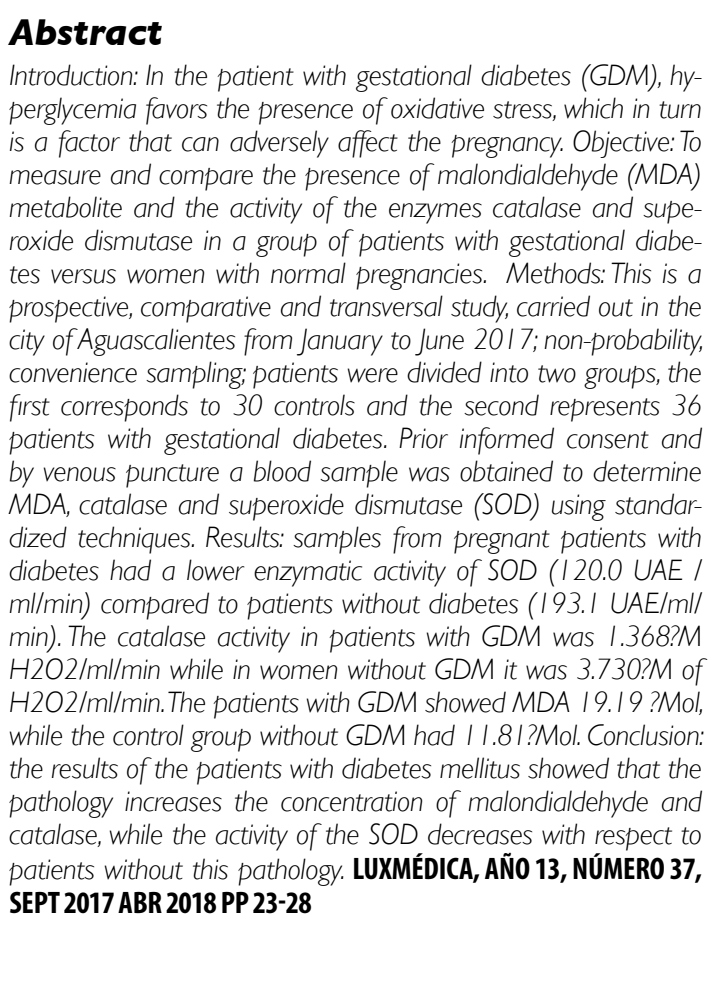 \\
\hline Palabr & oxidative stress, gestational diabetes \\
\hline
\end{tabular}

* Profesor investigador del Centro de Ciencias de la Salud de la Universidad Autónoma de Aguascalientes

** Profesor investigador del Centro de Ciencias Básica de la Universidad Autónoma de Aguascalientes

**** Investigadores del Instituto de Servicios de Salud del Estado de Aguascalientes

**** Técnica de laboratorio

***** Estudiantes del noveno semestre de la licenciatura de Análisis Químico Biológicos del Centro de Ciencias Básicas de la Universidad Autónoma de Aguascalientes

****** Estudiantes del noveno semestre de la licenciatura de Medicina del Centro de Ciencias de la Salud de la Universidad Autónoma de Aguascalientes

Fecha de recibido: 1 de diciembre 2017

Fecha de aceptación: 15 de enero 2018

Correspondencia: Alejandro Rosas Cabral, Edificio 107 planta alta. Campus Universitario. Avenida Universidad 940. Código postal 20131Aguascalientes, Ags., México. Teléfono 9108443 correo electrónico drrosascabral@gmail.com 


\section{Introducción}

La diabetes mellitus gestacional es un padecimiento caracterizado por la intolerancia a los carbohidratos con diversos grados de severidad, que se reconoce por primera vez durante el embarazo, y que puede o no resolverse después de éste. ${ }^{1}$ Su prevalencia a nivel mundial varía entre 4 y $19.6 \% .^{2-5}$ En México, aunque no existen estudios a nivel nacional, un estudio reciente reportó una prevalencia del $12.9 \% .^{6}$

El mecanismo patogénico predominante en la diabetes mellitus gestacional es la resistencia a la insulina relacionada con la presencia de hormonas como el lactógeno placentario. La DMG produce un incremento en el riesgo de resultados adversos perinatales a corto y a largo plazo. ${ }^{7}$

Se ha sugerido que las principales complicaciones en la DMG, incluidas las malformaciones congénitas, estén relacionadas con el estrés oxidativo; ${ }^{1,8,9}$ esta relación ya ha sido observada en modelo animal. Zhu y colaboradores sugieren que las mujeres con DMG desarrollan una resistencia a la insulina patológica y exhiben una disfunción de las células beta del páncreas, lo que les condiciona una menor habilidad para compensar el estrés oxidativo. ${ }^{10}$ Los sistemas antioxidantes maternos son importantes para proteger a las células embrionarias y fetales del potencial daño ocasionado por los metabolitos reactivos. ${ }^{11}$

El ambiente hiperglucémico e hiperlipémico que rodea al embrión y/o feto genera sustancias oxidantes con alta capacidad para dañar la estructura de las biomoléculas y alterar las funciones en que ellas participan. ${ }^{8}$ Recientemente Lappas y cols, publicaron que en las pacientes con DMG el estrés oxidativo, establecido por el incremento de radicales libres con una disminución de los sistemas antioxidantes, tiene implicaciones en la madre, en la función placentaria y en el bienestar fetal. ${ }^{7}$

Para identificar tempranamente a las mujeres con DMG, la Sociedad Americana de Diabetes en 2015, publicó la recomendación de realizar exámenes en busca de DMG entre las 24-28 semanas de gestación o antes, durante la primera visita prenatal, para aquellas que tengan factores de riesgo.

Debido al incremento observado en las últimas dos décadas de sobrepeso, obesidad y diabetes en nuestro país ${ }^{12}$, se espera un mayor número de mujeres embarazadas con factores de riesgo que favorezcan la presencia de la diabetes gestacional. El estrés oxidativo incrementado durante el embarazo asociado a diabetes gestacional puede conducir a repercusiones adversas durante la vida embrionaria y/o fetal, a corto, mediano y largo plazo. Por tales razones, en el presente trabajo nos propusimos como objetivo medir y comparar el nivel de estrés oxidativo en mujeres embarazadas con diagnóstico de diabetes mellitus gestacional vs controles de mujeres embarazadas sin diabetes gestacional. 


\section{Material y métodos}

Se realizó un estudio prospectivo, comparativo y transversal. Se estudiaron mujeres embarazadas con diagnóstico de diabetes mellitus gestacional, detectada durante las pruebas de tamizaje en centros de primer nivel de atención en la ciudad de Aguascalientes, de acuerdo con los criterios establecidos en la Guía de Práctica Clínica, Detección y Tratamiento de la Diabetes en el Embarazo de la Secretaría de Salud. Se utilizaron cualquiera de los siguientes cuatro criterios diagnósticos: glucemia en ayuno mayor o igual a $126 \mathrm{mg} / \mathrm{dL}$ en dos ocasiones, glucemia casual mayor de 200 $\mathrm{mg} / \mathrm{dl}$, prueba de tamiz con $50 \mathrm{~g}$ con resultado mayor o igual a $180 \mathrm{mg} / \mathrm{dl}$, o curva de tolerancia a la glucosa con $100 \mathrm{~g}$ o $75 \mathrm{~g}$ que cumpla con los criterios de Carpenter y Coustan o de la HAPO, respectivamente. Los criterios de inclusión fueron tener el diagnóstico de diabetes mellitus gestacional realizado en un centro de primer nivel de atención médica, con una edad mayor a 18 años y ser residente en la ciudad de Aguascalientes. Se excluyeron a las pacientes con diabetes mellitus diagnosticada previa al embarazo.

Se formaron 2 grupos de estudio: el primero conformado por mujeres con embarazo normal y el segundo conformado por mujeres embarazadas diagnosticadas con diabetes gestacional. La inclusión de las pacientes no fue probabilística, se realizó muestreo por conveniencia hasta completar 30 pacientes en cada grupo. El consentimiento informado de todas las participantes fue obtenido luego de la explicación verbal y escrita de los métodos, riesgos y beneficios contemplados en el estudio. El protocolo fue revisado y aprobado por los comités de ética del Instituto de Servicios de Salud del Estado de Aguascalientes y del Centro de Ciencias de la Salud de la Universidad Autónoma de Aguascalientes.

Muestras biológicas: de cada paciente se obtuvieron $5 \mathrm{ml}$ de sangre por punción venosa, sin anticoagulante. Las muestras se centrifugaron a $3000 \mathrm{rpm}$ durante 10 minutos, el sobrenadante (suero) se recuperó y fue almacenado a $-20^{\circ} \mathrm{C}$ hasta el momento de su análisis. Las variables estudiadas fueron: edad, peso, índice de masa corporal (IMC), escolaridad, antecedentes heredofamiliares, antecedentes personales patológicos, antecedentes personales no patológicos; y los siguientes estudios de laboratorio: glicemia, perfil de lípidos y nivel de estrés oxidativo en sangre. Las muestras biológicas se procesaron en el Laboratorio de Farmacología de la Universidad Autónoma de Aguascalientes.

\section{Determinaciones bioquímicas}

Se evaluó la actividad de las enzimas antioxidantes: enzima superóxido dismutasa (SOD) y catalasa (CAT); además se midió la concentración de indicadores de daño a biomoléculas como el malonildialdehído (MDA). Estos parámetros fueron determinados en suero mediante técnicas espectrofotométricas. Para medir la actividad de la SOD, se utilizó la técnica establecida por Marklund. Las muestras fueron leídas en el espectrofotómetro a $420 \mathrm{~nm}$. Para medir la actividad de la catalasa se utilizó la descomposición de $\mathrm{H} 2 \mathrm{O} 2$ a $240 \mathrm{~nm}$ a los 10 segundos y 70 segundos. La concentración sérica de productos de la lipoperoxidación fue determinada a través de la reacción colorimétrica de peróxidos lipídicos con ácido tiobarbitúrico (TBA) para dar malondialdehído (MDA) a $530 \mathrm{~nm}$. Se utilizó estadística descriptiva para el análisis de los datos sociodemográficos. Se comparó la media de los niveles de enzimas de estrés oxidativo entre ambos grupos por medio de pruebas paramétricas o no paramétricas (t de Student/U-Mann-Whitney) según su normalidad y la proporción de individuos en cada grupo con niveles enzimáticos considerados como estrés oxidativo (Chi cuadrada con prueba exacta de Fisher). Se utilizó el programa SSPS para el análisis estadístico. Se consideró significativo un valor de valor de $p$ de dos colas $\leq 0.05$ e intervalos de confianza del $95 \%$. 


\section{Resultados}

Quedaron incluidas 66 mujeres embarazadas. El grupo de pacientes con embarazo normal estuvo conformado por 30 mujeres y el grupo de diabéticas gestacionales por 36 mujeres. El promedio de edad fue de $22.7 \pm 5.2$ años (rango de 15 a 33 años) y de 28.2 \pm 8.4 años (con rango de 16 a 45 años) para el primero y segundo grupos, respectivamente $(p<0.002)$ En cuanto a peso corporal, encontramos que el promedio fue mayor en el grupo de diabetes gestacional, $79.4 \pm 14.2$ (rango de $58-110 \mathrm{Kg}$ ) comparado con el grupo de embarazo normal $66.4 \pm 12.6 \mathrm{Kg}$ (rango de 58 a $80 \mathrm{~kg}$ ) $(p<0.0002)$.

El índice de masa corporal fue mayor en el grupo de diabetes gestacional comparado con el grupo de embarazo normal, IMC $32.1 \pm 5.3$ vs $26.6 \pm 1.4(p<0.0001)$. La comparación de la talla y de la edad de inicio de vida sexual activa no mostró diferencias significativas (tabla 1 ). En el grupo de mujeres con diabetes gestacional, predominó la multiparidad.

\begin{tabular}{|c|c|c|c|}
\hline & $\begin{array}{c}\text { Grupo 1 } \\
\text { Embarazos normales } \\
\mathbf{n}=30\end{array}$ & $\begin{array}{c}\text { Grupo 2 } \\
\text { Diabetes gestacional } \\
\mathbf{n}=36\end{array}$ & $\mathbf{p}$ \\
\hline $\begin{array}{c}\text { Edad (años) } \\
\text { Promedio } \pm \text { desviación estándar }\end{array}$ & $22.7 \pm 5.2$ & $28.3 \pm 8.4$ & $0.002^{* *}$ \\
\hline $\begin{array}{c}\text { Peso }(\mathbf{k g}) \text { Promedio } \pm \text { desviación } \\
\text { estándar }\end{array}$ & $66.4 \pm 12.6$ & $79.4 \pm 14.2$ & $0.0002^{* * * *}$ \\
\hline $\begin{array}{c}\text { IMC (kg/m2) } \\
\text { Promedio } \pm \text { desviación estándar }\end{array}$ & $26.6 \pm 1.4$ & $32.1 \pm 5.3$ & $0.0001^{* * *}$ \\
\hline $\begin{array}{c}\text { Talla }(\mathbf{m}) \\
\text { Promedio } \pm \text { desviación estándar }\end{array}$ & $1.6 \pm 0.1$ & $1.6 \pm 0.1$ & - \\
\hline $\begin{array}{c}\text { IVSA (años) } \\
\text { Promedio } \pm \text { desviación estándar }\end{array}$ & $17 \pm 3.2$ & $18 \pm 4.3$ & - \\
\hline
\end{tabular}

Circunstancias comunes en ambos grupos fueron las siguientes: antecedente de diabetes en algún familiar en la mayoría de las pacientes, el estado civil predominante fue la unión libre, el nivel de escolaridad más frecuente fue la secundaria terminada y la ocupación de ama de casa.

Las muestras de las pacientes con diabetes gestacional tuvieron menor actividad enzimática de la SOD (120.0 UAE/ml/min) en comparación con las pacientes sin diabetes (193.1 UAE/ml/min) (figura 1). La actividad de la catalasa en las pacientes con DMG fue de $1.368 \mu \mathrm{M} \mathrm{H} 2 \mathrm{O} 2 / \mathrm{ml} / \mathrm{min}$ mientras que las mujeres sin DMG mostraron un valor de $3.730 \mu \mathrm{M} \mathrm{H} 2 \mathrm{O} 2 / \mathrm{ml} / \mathrm{min}$ (figura 2). Las pacientes con DMG mostraron un valor de $19.19 \mu \mathrm{Mol}$ de MDA, mientras que el grupo control sin DMG tuvo un valor de $11.81 \mu \mathrm{Mol}$.

\begin{tabular}{|c|c|c|c|}
\hline & $\begin{array}{c}\text { Grupo 1 } \\
\text { Embarazos normales } \\
\mathrm{n}=30\end{array}$ & $\begin{array}{c}\text { Grupo 2 } \\
\text { Diabetes gestacional } \mathrm{n}=36\end{array}$ & $\mathrm{p}$ \\
\hline $\begin{array}{c}\text { Superóxido dismutasa } \\
(\mathrm{UAE} / \mathrm{ml} / \mathrm{min})\end{array}$ & 193.1 & 120.0 & $0.001^{*}$ \\
\hline $\begin{array}{c}\text { Catalasa } \\
\mu \mathrm{M} \mathrm{H2O2/ml/min}\end{array}$ & 3.730 & 1.368 & $0.001^{*}$ \\
\hline $\begin{array}{c}\text { Malondialdehído } \\
\mu \text { Mol }\end{array}$ & 11.81 & 19.19 & 0.25 \\
\hline
\end{tabular}




\section{Discusión}

El estrés oxidativo puede definirse como el desbalance entre la producción de especies reactivas de oxígeno y las defensas del sistema antioxidante, el cual puede ocasionar diversos cambios que al final producen daño y/o muerte celular. Así pues, el estrés oxidativo puede provenir de un incremento en la cantidad de especies reactivas de oxígeno producidas o en una disminución de las enzimas defensoras que las degradan. ${ }^{7}$

El estrés oxidativo ha sido claramente asociado a la diabetes mellitus tipo 2 , donde se encuentran disminuidas las defensas antioxidantes de las enzimas como la catalasa, la superóxido dismutasa y glutatión peroxidasa aunado a la inhibición de la glutatión reductasa debido a la hiperglicemia, lo que condiciona un incremento de las especies reactivas de oxígeno (ERO). ${ }^{13-15} \mathrm{El}$ daño a los lípidos de las membranas celulares está reflejado con el incremento de la lipoperoxidación y sus productos finales como el malondialdehido. ${ }^{16}$

En este estudio, encontramos incrementada la concentración sérica del marcador bioquímico de daño oxidativo: malondialdehido, producto final de la lipoperoxidación ocasionada por la presencia de metabolitos reactivos del estrés oxidativo que acompaña a la diabetes gestacional. Este hallazgo ha sido reportado por varios autores que han propuesto que los radicales libres pueden reaccionar con los lípidos de las membranas celulares y ocasionar daño celular en las pacientes con diabetes gestacional. Shang y cols. (2015) realizaron un estudio para comparar el nivel de estrés oxidativo en mujeres diagnosticadas con DMG, midiendo el estrés oxidativo a través de malondialdehído, 8-isoprostano, xantina oxidasa, peróxidos lipídicos, superóxido dismutasa, glutatión peroxidasa, capacidad total antioxidante, vitamina $C$ y vitamina $E$ en plasma de la madre, plasma del cordón y en placenta. Demostrando un incremento significativo en malondialdehído, xantina oxidasa y 8 isoprostano y decremento significativo de superóxido dismutasa y capacidad total antioxidante en las mujeres con DMG con respecto a un grupo control, datos similares a los encontrados en este trabajo. ${ }^{17}$

El desequilibrio entre la formación y la inactivación de radicales libres de oxígeno o de metabolitos reactivos, también produce lesión a las macromoléculas celulares proteicas como el DNA. Por otro lado, es sabido que las vías enzimáticas antioxidantes, representadas en este estudio por la superóxido dismutasa y la catalasa, protegen del daño celular, y se ha asociado la disminución de la capacidad antioxidante y el incremento de la lipoperoxidación a la diabetes gestacional. ${ }^{16}$

Se ha sugerido que el principal factor para el incremento del estrés oxidativo y la disminución en la actividad de la catalasa en mujeres con DMG es el ambiente hipoglucémico gestacional, el cual cambia el balance REDOX e interfiere sobre la actividad de la gamma aminolevulínico deshidratasa. ${ }^{18}$ En el 2003, Orhan y colaboradores reportaron una menor actividad de catalasa en suero de mujeres con DMG y con diabetes mellitus dependiente de insulina. ${ }^{19}$ De la misma manera, Visiedo y cols. reportaron menor actividad de catalasa en placentas de mujeres con DMG. ${ }^{20}$ Nosotros también encontramos una disminución significativa de la actividad de la catalasa en nuestras pacientes con diabetes gestacional comparado al de las pacientes con embarazos normales. En cuanto a la SOD, algunos autores ${ }^{21-22}$ han reportado una menor actividad de la SOD en las mujeres con DMG, dato similar a lo encontrado por nosotros.

Se ha considerado que la paciente que padeció diabetes gestacional tiene un riesgo mayor de desarrollar síndrome metabólico, diabetes mellitus tipo 2 y enfermedad cardiovascular en etapas posteriores de su vida. ${ }^{16}$ Esto resalta la importancia de implementar medidas que disminuyan la posibilidad de desarrollo de DMG en nuestra población y que esta patología sea la desencadenante de mayor morbilidad y riesgo de 
mortalidad en las mujeres de nuestro país. Tal es el caso de lo reportado por Maged y colaboradores, al administrar un gramo de ácido ascórbico diario versus placebo a dos grupos de mujeres con diabetes mellitus gestacional, notando una disminución en la necesidad de administrar insulina para el control de la glicemia en las mujeres a las que se les administró ácido ascórbico, al igual que un incremento en la actividad de SOD y catalasa y un descenso en la cifra de MDA en sangre y en placenta. ${ }^{21}$

En nuestras pacientes encontramos diferencias significativas cuando comparamos

\section{Bibliografía}

1. Acevedo, C; López, F; Sepúlveda, S; Espinosa, V (2007) Actividad de la glutatión reductasa en el embarazo diabético. Revista Chilena de Obstetricia y Ginecología; 72(2):82-88

2. Duarte, GM; Muñoz, G; Rodríguez, S; et al (2004) Prevalencia, detección y tratamiento de la Diabetes Gestacional. Revista de Salud Pública y Nutrición.

3. Setji, TL; Brown, AJ; Feinglos, MN (2005) Gestational Diabetes Mellitus. Clinical Diabetes, 23(1):17-24

4. Ramírez M (2005) Diabetes Mellitus Gestacional: Experiencia en una institución de tercer nivel de atención. Ginecología y Obstetricia de México; 73:484:491

5. Ríos-Martínez, W; García-Salazar, AM Ruano-Herre$\mathrm{ra}$, L; et al (2014) Complicaciones obstétricas de la diabetes gestacional: criterios de la IADPSG y HAPO Perinatologíay Reproducción Humana; 28(1):27-32

6. Ramírez-Torres MA. (2013) The importance of gestational diabetes beyond pregnancy. Nutr Rev; 71: Suppl 1: S37-41

7. Lappas M, Hiden U, Desoye G, Froehlich J, Hauguel-de Mouzon S, Jawerbaum A (2011) The role of oxidative stress en the pathophysiology of gestational diabetes mellitus. Antioxid Redox Signal; 15(12):3061-3100.

8. Fernández Romero, T; Clapés Hernández, S; Suárez Román, G; et al (2010) Marcadores de estrés oxidativo en embarazadas diabéticas. Revista Cubana de Investigaciones Biomédicas; 29(4).

9. García, D; García, R (2009) Avances en la patogénesis de la embriopatía diabética. Revista Médica de Chile; 137:1627-1635.

10. Zhu C, Yang $H$, Geng $Q$, Ma Q, Long $Y$, Zhou $C$, Chen $M$ (2015) Association of oxidative stress biomarkers with gestational diabetes mellitus in pregnant women: a case-control study. Plos One; 27(10): 4 .

11. Eren R, Aykal G, Sayrac S, Erol O, Ellidag HY, Yilmaz N. (2017) Relationship between thioredoxin and thioredoxin-binding protein in patients with gestational diabetes mellitus. J Matern Fetal Neonatal Med; 30(2):164-168. los promedios de la edad, el peso corporal y el índice de masa corporal entre los dos grupos, lo que refleja la importancia de recomendar en la consulta preconcepcional posponer el embarazo hasta tener un IMC acorde a un peso normal.

En conclusión, nosotros encontramos un incremento en la producción de sustancias oxidantes expresadas mediante la concentración de MDA, aunado a una disminución en la actividad de enzimas antioxidantes medidas como actividad de catalasa y SOD en suero de mujeres con DMG en comparación con mujeres con embarazo normal.

12. Gutiérrez JP, Rivera Dommarco J, Shamah Levy (2012) Encuesta Nacional de Salud y Nutrición 2012 Resultados Nacionales, México: Instituto Nacional de Salud Pública.

13. Loeken, $M$ (2006) La hiperglucemia materna induce defectos fetales neurológicos por aumento del estrés ooxidativo. Journal of the Society for Gynecologic Investigation; 13(1):2-10.

14. Ramos Ibarra, ML; Batista González, CM; Gómez Meda, BC; Zamora Pérez, AL (2006) Diabetes, estrés oxidativo y antioxidantes. Investigación en salud; 8(1):7-15

15. Calderón Salinas, JV; Muñoz Reyes, EG; Quintana Escorza, MA (2013) Estrés oxidativo y Diabetes Mellitus. Revista de Educación Bioquímica; 32(2):53-66.

16. Parast VM. (2017) Antioxidant status and risk of gestational diabetes mellitus: a case-control study. Clin Nutr Res; 6(2):81-88.

17. Shang, M; Zhao, J; Yang, L; Lin, L (2015) Oxidative stress and antioxidant status in women with gestational diabetes mellitus diagnosed by IADPSG criteria. Diabetes Research Clinical Practice; 109(2):404 410.

18. Rodrigues F. (2017) Influence of gestational diabetes in the activity of delta-aminolevulinic dehydratase and oxidative stress biomarkers. Redox Rep; 17:1-5.

19. Orhan H (2003) Circulating biomarkers of oxidative stress in complicated pregnancy. Arch Gynecol Obstet; 7(4):189-195.

20. Visiedo $F$ (2017) Characterization of no-induced nitrosative status in human placenta from pregnant women with gestational diabetes mellitus. Oxid Med Cell Longev, 5629341

21. Maged AM, Torky $H$, Fouad MA, Gadallah SH, Waked NM, Gayed AS, Salem AK (2016) Role of antioxidants in gestational diabetes mellitus and relation to fetal outcome: a randomized controlled trial. J Matern Fetal Neonatal Med; 29(24): 4049-4054.

22. Mordwinkin NM, Ouzounian JG, Yedigrova L, Montoro MN, Louie SG, Rodgers KE. (2013) Alteration of functional markers in women with gestational diabetes and their fetuses. J Matern Fetal Neonatal Med; 5:507-12 